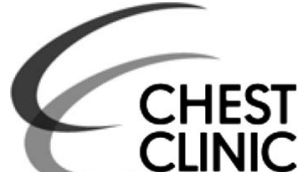

${ }^{1}$ Respiratory Institute,

Cleveland Clinic, Cleveland, Ohio, USA

${ }^{2}$ Anesthesiology Institute, Cleveland Clinic, Cleveland, Ohio, USA

${ }^{3}$ Pathology and Laboratory Medicine Institute, Cleveland Clinic, Cleveland, Ohio, USA

\section{Correspondence to} Dr Chris Lau, Respiratory Institute, Cleveland Clinic, 9500 Euclid Ave, Cleveland, OH 44195, USA:

lauc@ccf.org

Received 11 March 2016 Revised 19 May 2016 Accepted 20 May 2016 Published Online First 10 June 2016

\title{
Whole lung lavage for lipoid pneumonia
}

\author{
Chris Lau, ${ }^{1}$ Basem B Abdelmalak, ${ }^{2}$ Carol F Farver, ${ }^{3}$ Daniel A Culver ${ }^{1}$
}

We evaluated a 49-year-old woman who had worsening shortness of breath, non-productive cough and recurrent low-grade fevers over 6 months despite several courses of antibiotics. Her medical history was remarkable for suspected immunodeficiency that had been treated with intravenous immunoglobulin and recurrent pneumonia, including methicillin-resistant Staphylococcus aureus empyema requiring left decortication 2 years earlier. Repeated CT scans of her lung showed progressive patchy ground-glass and consolidative opacities bilaterally with septal prominence (figure 1A).

We performed bronchoscopy. Bronchoalveolar lavage cultures and special stains for bacteria, acid-fast bacilli and fungi were negative. Transbronchial biopsies revealed clusters of alveolar macrophages with lipid vacuoles, consistent with lipoid pneumonia. Periodic acid Schiff stains were negative (figure 2). Although no definite source for lipid inhalation was identified, she admitted to coating her nares during the winter with petroleum jelly since her empyema 2 years prior.

Despite removal of the suspected agent and treatment with corticosteroids, she had continued to decline over a 2-month follow-up period. Her oxygen requirements increased to $4 \mathrm{~L} / \mathrm{min}$ and she reported worsening exertional dyspnoea; pulmonary function testing and imaging continued to deteriorate.

We performed bilateral whole lung lavage using a double lumen tube under general anaesthesia as previously described. ${ }^{1}$ We used aliquots of prewarmed normal saline to a total of 16 and $21 \mathrm{~L}$ for the right and left lung, respectively. After each aliquot, the lung was allowed to drain using a chest percussion device. The initial lavage return was turbid, with striking yellow fat globules floating in the supernatant in contradistinction to pulmonary

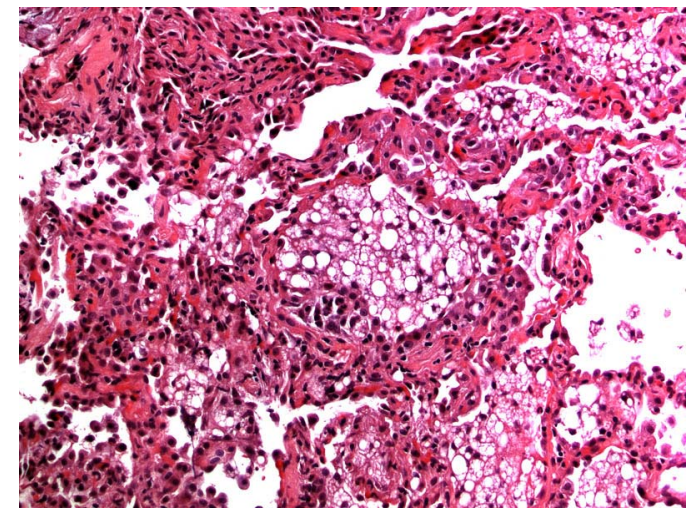

Figure 2 Foamy lipid-laden histiocytes filling alveolar spaces with surrounding reactive pneumocytes confirming the diagnosis of lipoid pneumonia (H\&E; 400x).

alveolar proteinosis (PAP), where the higher density lipoproteinaceous material is expected to settle to the bottom. Subsequent lavages were clearer such that by the final lavage, the returned fluid was clear (figure 3).

At outpatient follow-up the patient reported significant clinical improvement, including resolution of her cough and fevers. Her oxygen requirements normalised and her pulmonary function testing returned to her previous baseline. Repeat CT of the lungs also showed dramatic improvement (figure 1B).

Lipoid pneumonia is classified into exogenous and endogenous subtypes based on the source of exposure. Exogenous lipoid pneumonia is typically related to aspiration of oil-based substances such as petroleum jelly and mineral oils. ${ }^{2}$ The clinical presentation is nonspecific, varying widely depending on the duration and amount of oil aspirated. Common symptoms include chronic cough and dyspnoea; fever,
To cite: Lau $C$,

Abdelmalak $B B$, Farver $C F$, et al. Thorax

2016;71:1066-1067.

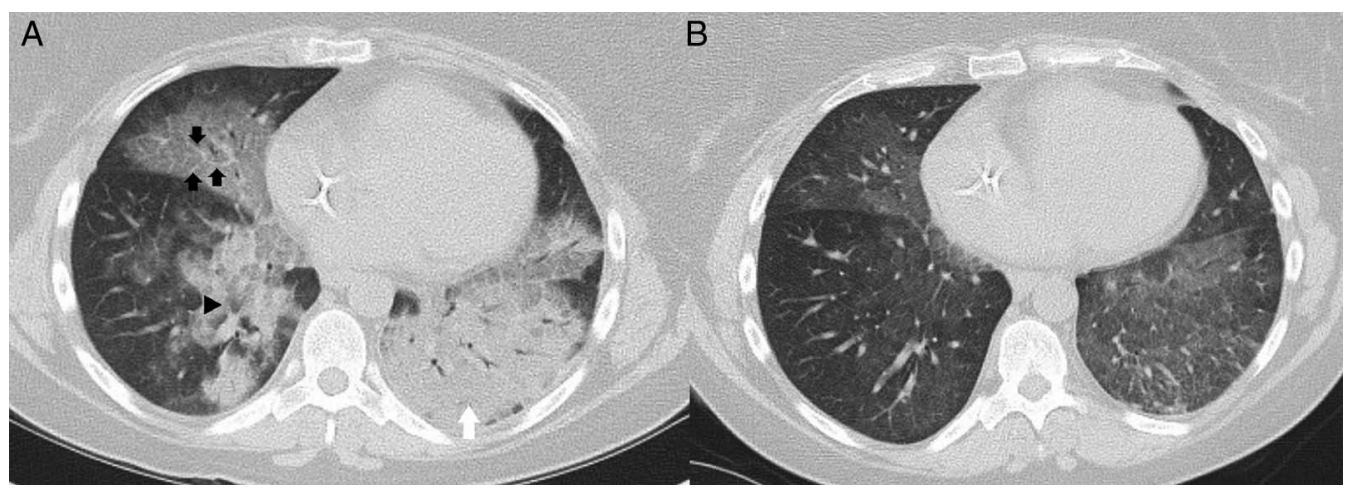

Figure $1 \mathrm{CT}$ of the lung showing (white arrow) consolidation with (black triangle) patchy ground-glass opacities and (black arrows) septal thickening $(A)$ before and (B) after whole lung lavage. 

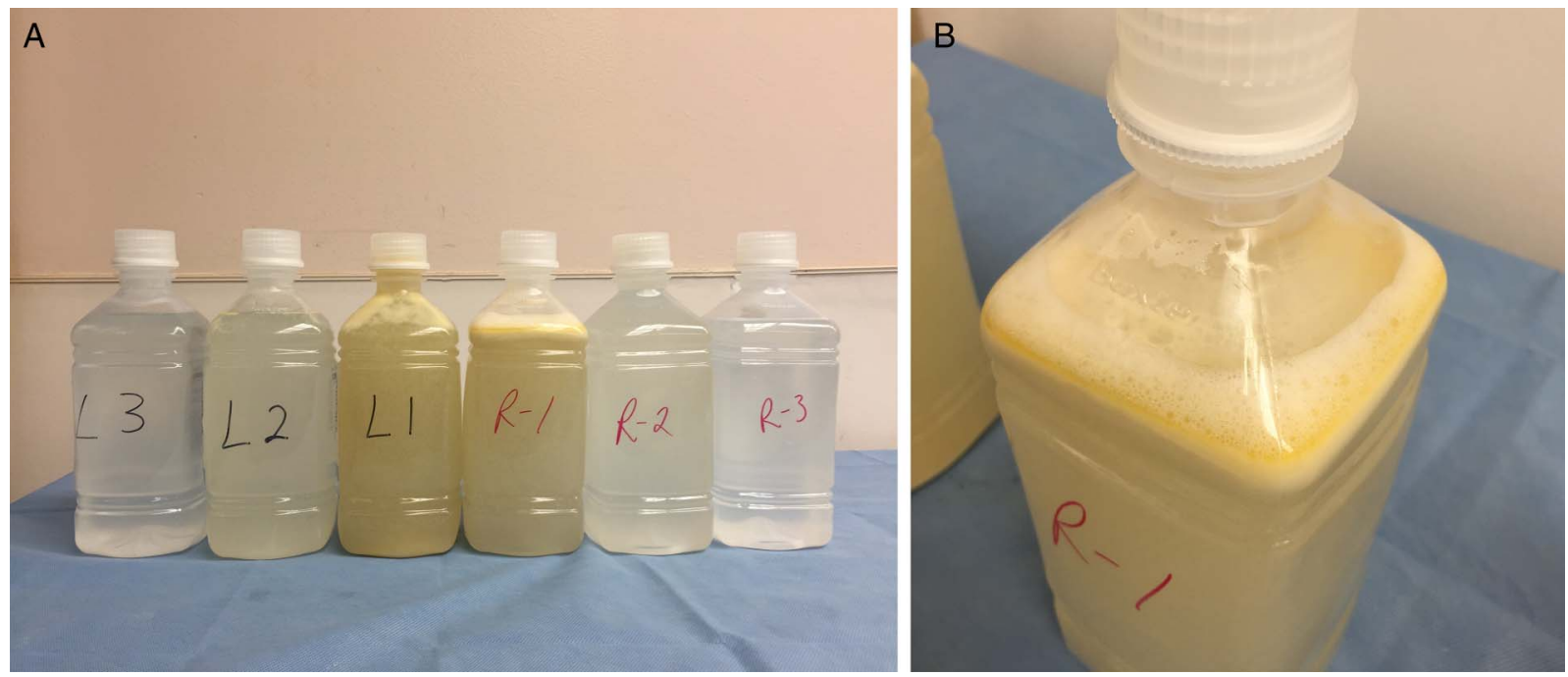

Figure 3 Aliquots of normal saline from whole lung lavage. (A) Consecutive aliquots from the left and right lung showing improvement of return lavage. (B) Floating oil-based supernatant from first right lung aliquot.

chest pain and haemoptysis are less frequent. ${ }^{2}$ The CT appearance is also highly variable, including airspace consolidation, groundglass opacities, and sometimes a 'crazy-paving' pattern. Diagnosis is difficult, as it requires a high degree of suspicion and thus is often delayed. Suspicion should arise whenever CT imaging shows a chronic unrelapsing pattern of ground-glass opacities with 'crazypaving'. Treatment usually entails removal of the offending agent. There have been anecdotal reports suggesting that corticosteroids may be useful. Whole lung lavage, typically reserved for PAP, ${ }^{1}$ has been reportedly successful in two adult cases of exogenous lipoid pneumonia. ${ }^{2}$ Our case highlights whole lung lavage as an underrecognised therapy for refractory lipoid pneumonia, but it should only be considered in experienced centres.
Contributors $\mathrm{CL}, \mathrm{BBA}$ and $\mathrm{DAC}$ managed the patient. $\mathrm{CL}$ prepared the manuscript. $\mathrm{CL}$ and CFF prepared the images. BBA, CFF and DAC revised the manuscript.

Competing interests None declared.

Patient Consent Obtained.

Provenance and peer review Not commissioned; externally peer reviewed.

\section{REFERENCES}

1 Abdelmalak BB, Khanna AK, Culver DA, et al. Therapeutic whole-lung lavage for pulmonary alveolar proteinosis - a procedural update. J Bronchol Intervent Pulmonol 2015;22:251-8.

2 Chang HY, Chen CW, Chen CY, et al. Successful treatment of diffuse lipoid pneumonitis with whole lung lavage. Thorax 1993;38:947-8.

3 Wong CA, Wilsher ML. Treatment of exogenous lipoid pneumonia by whole lung lavage. Aust N Z J Med 1997;165:128-31. 